\title{
Publisher Correction: Is wave-particle objectivity compatible with determinism and locality?
}

Radu Ionicioiu, Thomas Jennewein, Robert B. Mann \& Daniel R. Terno

Nature Communications 5:4997 doi: 10.1038/ncomms5997 (2014); Published online 26 Sep 2014; Updated 26 Mar 2018

The original HTML version of this Article had an incorrect article number of 3997; it should have been 4997. This has now been corrected in the HTML; the PDF version of the Article was correct from the time of publication. 